\title{
The transcrestal hydrodynamic ultrasonic cavitational sinuslift: Results of a 2-year prospective multicentre study on 404 patients, 446 sinuslift sites and 637 inserted implants
}

\author{
Angelo Troedhan ${ }^{1}$, Andreas Kurrek ${ }^{2}$, Marcel Wainwright ${ }^{3}$, Izabela Schlichting ${ }^{1}$, \\ Bianca Fischak-Treitl ${ }^{4}$, Martin Ladentrog ${ }^{5}$ \\ ${ }^{1}$ Center for Facial Aesthetics Vienna, Vienna, Austria \\ ${ }^{2}$ Implantology Clinic Oberkassel, Dusseldorf, Germany \\ ${ }^{3}$ Implantology Clinic Kaiserswerth, Dusseldorf, Germany \\ ${ }^{4}$ Office for General Dentistry and Implantology, Mattersburg, Austria \\ ${ }^{5}$ Office for General Dentistry and Implantology, Graz, Austria \\ Email: troed@aon.at
}

Received 7 October 2013; revised 11 November 2013; accepted 23 November 2013

Copyright (C) 2013 Angelo Troedhan et al. This is an open access article distributed under the Creative Commons Attribution License, which permits unrestricted use, distribution, and reproduction in any medium, provided the original work is properly cited.

\begin{abstract}
Introduction: In 2006 an ultrasound-surgery-based method to hydrodynamically detach the sinus-membrane utilizing the ultrasonic cavitation effect-the tHUCSL - was developed and a surgical protocol established. The aim of the study was to determine the indication-range and success-rate of this novelty procedure. Materials \& Methods: Between 2007 and 2009, 404 patients were treated by 6 oral surgeons of different experience-levels with the tHUCSL in 446 sinussites. 637 implants were inserted and then prosthodontically treated and observed and documented until December 2011. The subantral space was augmented via the $3 \mathrm{~mm}$ transcrestal approach with an augmentation volume of $1.9 \mathrm{ccm}(+/-0.988 \mathrm{ccm})$ and an augmentation height of $10.7 \mathrm{~mm}(+/-2.85 \mathrm{~mm})$. Results: Within the survey-period $15(2.35 \%)$ of the 637 inserted implants were lost, mostly before implant loading due to postsurgical infection and nonosseointegration in the augmentation site. 1 implant was lost after implant loading and prosthetic treatment within 1 year after loading. The overall success rate with functional implants in site is $97.65 \%$ evenly distributed among the participating surgeons. $86 \%$ of the patients were observed with no postsurgical swelling and $87 \%$ no postsurgical pain. Discussion: The results suggest the tHUCSL to be a safe minimal-invasive alternative to traditional lateral approach and transcrestal osteotome sinuslift-procedures applicable to all anatomical situations.
\end{abstract}

Keywords: Transcrestal; Hydrodynamic Sinuslift; Bone Augmentation; Implants; Ultrasound Surgery; Maxillary Sinus

\section{INTRODUCTION}

The basic principle of subantral bone augmentation ("Sinuslift") in the lateral maxilla is a commonly accepted, well documented and established procedure for bone augmentation in the posterior maxilla to allow implant insertion in the atrophic maxillary alveolar crest since the 80's of the past century. Various surgical techniques have been developed, described and scientifically evaluated over the centuries with clinically good results [1-10].

Various authors published different results and preferences on the grafting material subantrally inserted (autologous, heterologous, xenogenic, allogenic bone, synthetic bone grafts) [11-19]. Nevertheless a survey over the current literature and systematic reviews [20] suggests the success of sinus floor augmentation procedures to be related more to the medical history of the augmented sinus and the skills of the surgeon than the used bone graft material [21].

The success of bone augmentation procedures especially in sinus-floor augmentation can be considered scientifically proven as to be more related to intact anatomical and physiological structures of the periosteum and a sufficient blood supply of the augmentation site than to any specific bone graft material [22-26]. 
Diligent presurgical diagnosis, intrasurgical complication management and postsurgical patient and complication management can be considered to play a major role in the overall success of subantral augmentation procedures [27-31] as well as the experience of the surgeon as clinical research in other fields of oral surgery suggests $[32,33]$. The more bone augmentation surgery is performed with highly invasive surgical methods, the more postsurgical morbidity can be expected as voluminous edema, pain, uncontrollable bleeding and long term failure of the augmentation.

Proper education and training of the oral surgeon are mandatory to develop the necessary skills for traditional sinuslift-procedures with lateral and/or transcrestal access to prevent the most feared intrasurgical complication of a partial or entire rupture of the sinus-membrane.

Therefore, sinuslift procedures also follow the trend from massive invasive surgical protocols with a lateral approach and lateral window preparation [1-3] towards minimal invasive ultrasound surgical techniques [34] based on the idea of the minimal invasive transcrestal approach [4,5]. The limited possible augmentation volume of the classical Summers lift was partly enhanced by the introduction of balloon and hydraulic pressure assisted transcrestal sinuslift techniques [35-37].

Still these procedures are challenging the manual diligence of the performing, surgeon and proper training and experience have to be achieved by the surgeon to perform these techniques with an acceptable success rate. Above all-in case of failure-the inconveniences to the patient (edema, pain, costs, long term morbidity) have to be kept as small as possible.

To combine the advantages of a lateral approach sinuslift (scalable augmentation area and volume) with the atraumaticity of the transcrestal approach and to avoid the need of the surgeon to touch the sinus membrane with instruments or balloons in the detachment-process (and therewith eliminate one of the major risks of intrasurgical membrane perforation) and to utilize the well documented advantages of hydrodynamic pressure sinusmembrane-detachment [36,37] and atraumaticity of ultrasonic surgery, the transcrestal hydrodynamic ultrasonic cavitational sinuslift (tHUCSL-INTRALIFT) was developed in 2006 [38-40].

The significant application safety of the tHUCSLINTRALIFT compared to traditional transcrestal Summers- and balloon-assisted sinuslift-procedures can be considered as verified in animal cadaver experiments [41].

The aims of the current prospective multicentre study performed in six implantologist's offices were:

1) The evaluation if success-rates of the tHUCSLINTRALIFT depend on the experience-level of the performing oral surgeon, the type and/or brand of suban- trally inserted bone graft material and type and/or brand of inserted dental implants.

2) The verification of the unrestricted applicability of the tHUCSL-INTRALIFT to every subantral anatomical surgical site from very narrow single tooth-gaps up to bilateral endentolous upper jaws and the vastly varying anatomy of the human maxillary sinus as suggested by the experimental results [38].

3) The evaluation of clinical application safety as suggested by comparative animal cadaver studies [41] when long term experienced implantologists are compared to average experienced ones.

4) The evaluation of the procedural burdening of patients such as postsurgical pain and swelling and overall success-rate of the tHUC-sinuslift-technique.

\section{MATERIAL \& METHODS}

\subsection{Experience Level of Participating Surgeons}

To be as close as possible to working conditions of most oral surgeons, 6 dentists in 5 offices were participating in the prospective study treating 412 patients in 457 maxillary sinus sites with a subantral alveolar crest height of 1 - $10 \mathrm{~mm}$ with the tHUCSL-INTRALIFT procedure between February 2007 and December 2009. (Although subantral crest heights of 8 - $10 \mathrm{~mm}$ might be treated with short implants nowadays the authors decided the inclusion when the protocol was setup in 2006 due to lack of documented long-term success rates of short implants in the molar region of the maxilla at that time.)

One participating dentist is also specialized in oromaxillofacial surgery for 20 years, three dentists have long term experience in implantology and sinuslifting for 5 - 10 years, one participating dentist short time experience in implantology for 4 years and one dentist was at the beginner level in implantology with 2 years of practical experience, the latest two without experience in sinuslifting.

\subsection{Patients Inclusion Criteria}

To be eligible for tHUC-sinuslift surgery all patients had to fulfil the common criteria for any type of sinusliftprocedure (no prior Caldwell-Luc-Sinus-surgery, no chronic or acute maxillary sinusitis, no acute cold, no mucocele visible in panoramic X-ray, permission by General Practitioner/Internist/ENT). Smokers were considered as eligible since percentage of smokers in the European population is still high with an average of $30 \%$. All patients requesting a sinuslift in all 5 participating clinics/offices within the study period and fulfilling the common eligibility-criteria for any sinuslift procedure were exclusively treated with the tHUCSL-Intralift procedure. 


\subsection{Surgical Protocol}

The tHUCSL-INTRALIFT procedure had to be performed according to the strict surgical protocol with Piezotome I/II/SOLO or Implant Center I/II (SatelecACTEON/FR) set up by the developers of the tHUCSLIntralift presented in their research protocol [38] and documented by pre-, post-surgical and follow-up calibrated digital panoramic $\mathrm{x}$-ray:

The alveolar crest had to be revealed by either a $6 \mathrm{~mm}$ diameter mucoperiostal punch (Figure 1) or a minimal invasive crestal mucoperiostal flap not or only slightly exceeding the occlusal surface of the alveolar crest (Figure 2).

The transcrestal approach then had to be marked with the TKW 1 diamond coated conical ultrasound tip (Figure 3) followed by the opening of the cortical bone of the sinus floor with the TKW 2 tip which has a diameter of $2.4 \mathrm{~mm}$. (Figures 4 and 5). A receptacle for

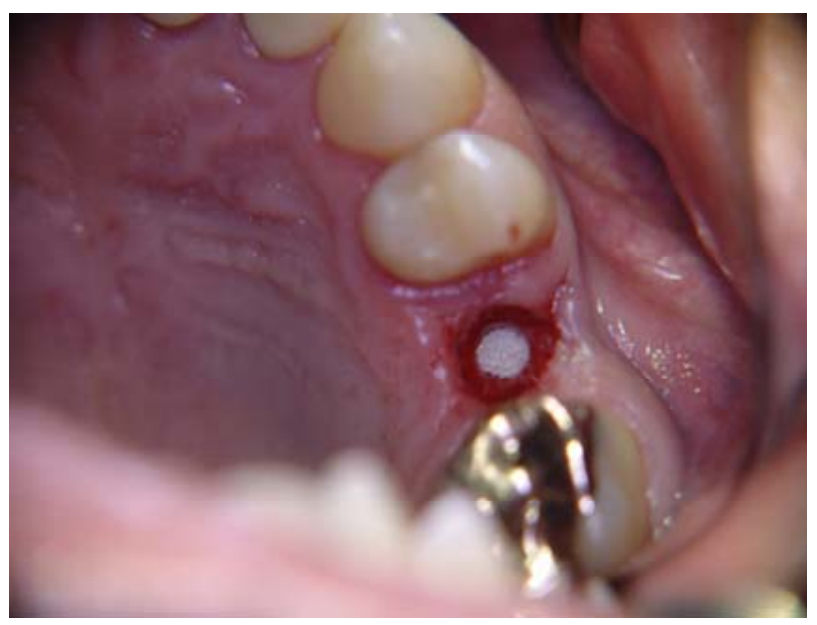

Figure 1. tHUCSL-INTRALIFT crestal approach via a $6 \mathrm{~mm}$ gingival punch.

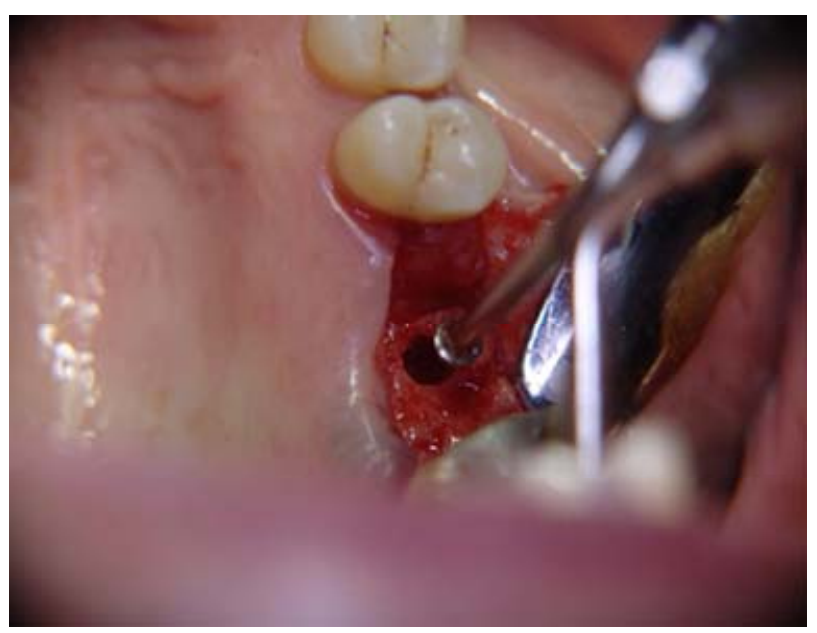

Figure 2. tHUCSL-INTRALIFT crestal approach via a topcrestal mucoperiostal flap.

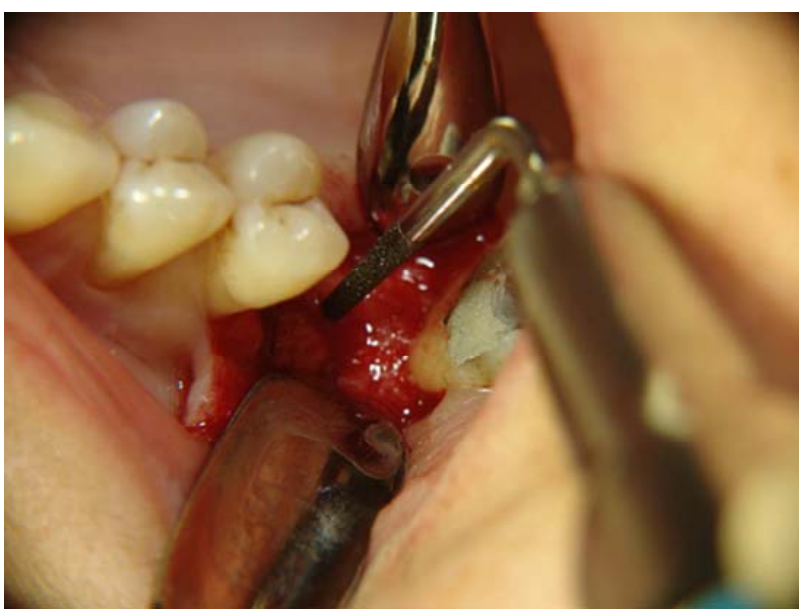

Figure 3. TKW 1 diamond coated conical tip for Piezotome I/ II/SOLO.

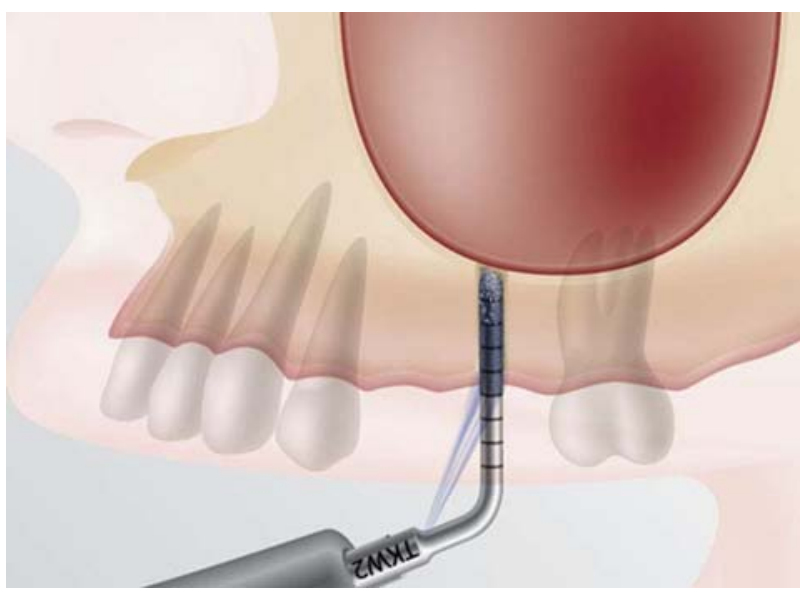

Figure 4. Opening of the bony sinusfloor with the cylindrical and rounded diamond coated TKW 2 tip-schematic.

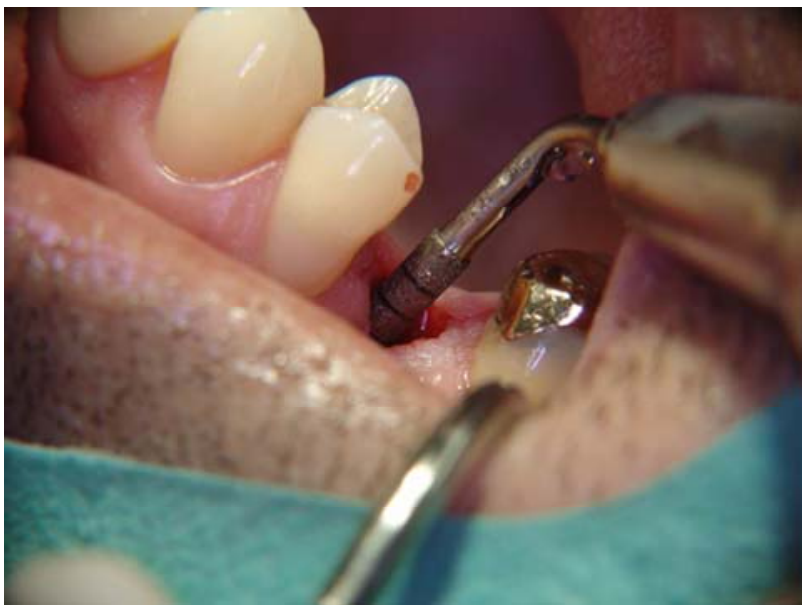

Figure 5. TKW 2 tip inserted to open the sinusfloor.

the hydrodynamic ultrasonic applicator TKW 5 (diameter $3.0 \mathrm{~mm}$ ) had to be prepared with TKW 4 tip, which has a diameter of $2.8 \mathrm{~mm}$ (Figure 6). 


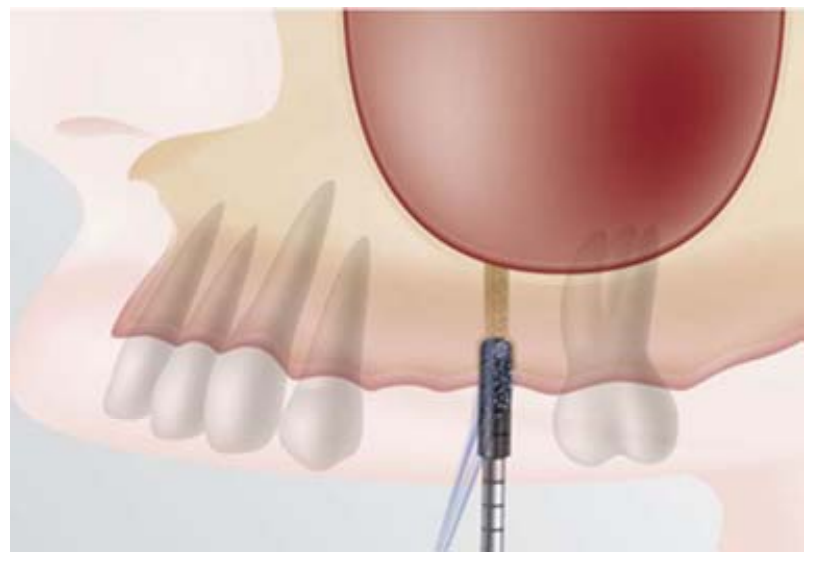

Figure 6. Preparation of the $2.8 \mathrm{~mm}$ receptacle with the cylindrical diamond coated TKW 4-tip-schematic.

After checking the snug fit of the TKW 5-applicator in the receptacle the hydrodynamic ultrasonic cavitational detachment of the sinus membrane had to be performed at a power setting of the Piezotome at mode D3 with a flow rate of $30 \mathrm{ml} / \mathrm{min}$ for 5 seconds. At these settings the sinus membrane will be completely detached from the entire sinus floor and provides a possible augmentation volume of $2.5 \mathrm{ccm}$. (Figures 7 and 8 )

The integrity of the sinus-membrane had to be checked visually and/or by Valsalva-test. In case of a perforation of the sinus-membrane the surgeon had to decide wether to proceed or interrupt surgery for wound closure depending on the size of the perforation when visible.

After widening of the transcrestal trepanation with TKW 3 (Figure 9) and TKW 4 diamond coated ultrasound tips (Figure 10) synthetic, xenogenic and/or autologous bone graft of the surgeon's choice was inserted under the sinus membrane with a common bone applicator (Figure 11).

When available the surgeons were advised to check the augmentation area and height intraoperatively step by step with digital $\mathrm{X}$-ray to determine the case-sufficient extension of the augmentation. (Figures 12-14)

It was also the surgeon's decision to insert one or more implants simultaneously depending on the height and quality of the subantral alveolar crest to achieve primary stability with the surgeon's common implant system. The only prerequisite towards the choice of the implant was an implant-diameter wider than $3 \mathrm{~mm}$ since the diameter of the TKW 5 tip is $3 \mathrm{~mm}$.

\subsection{Study Data Documented and Follow Up}

Data as follows then had to be documented by the participating dentists in a web-based Excel-Sheet accessible via password:

Date of surgery, name of the surgeon coded by a number, patient's name, age, sex, smoker $\mathrm{y} / \mathrm{n}$, diabetes

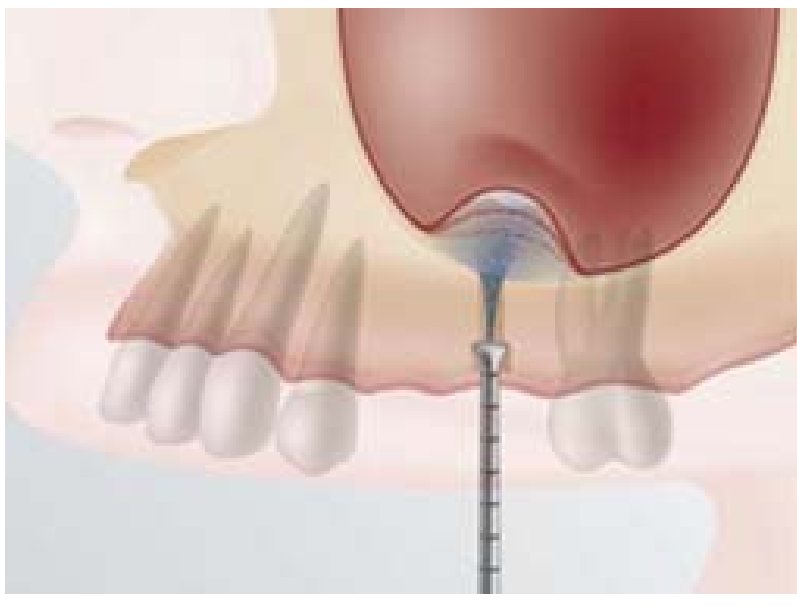

Figure 7. Insertion of the hollow $3.0 \mathrm{~mm}$ TKW 5-tip and detachment of the sinus-membrane by injection of ultrasonic oscillating saline solution creating a cavitation effect—schematic.

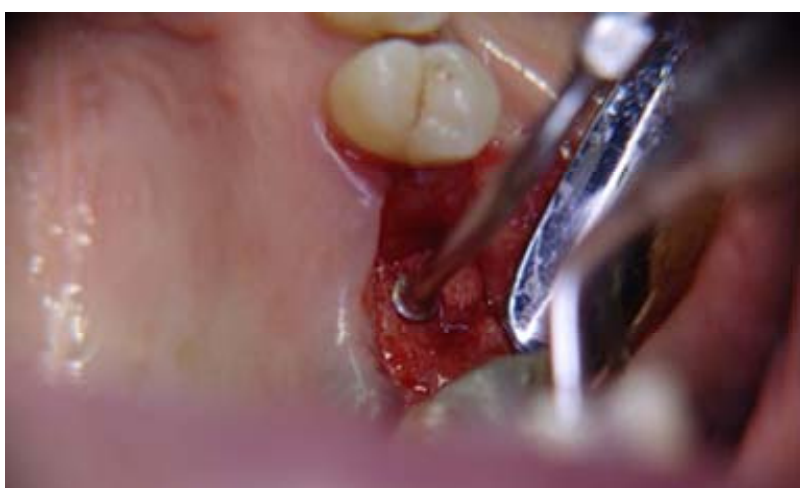

Figure 8. TKW 5-tip inserted into the receptacle: this tip seals the approach canal like a sealing ventile and enables by it's oscillations the cavitation effect.

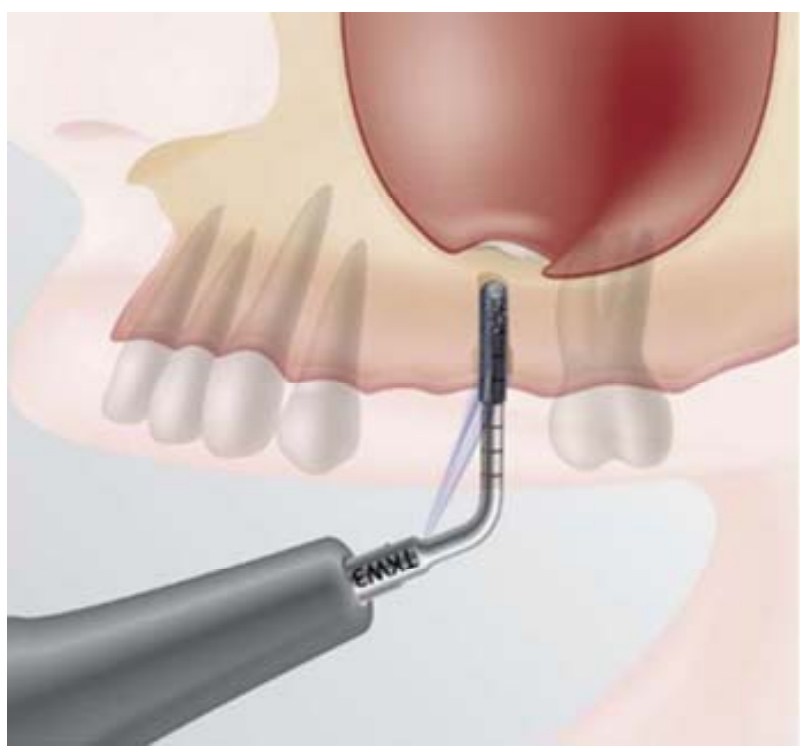

Figure 9. After detachment of the sinus-membrane from the bony floor the approach canal is widened with the cylindrical diamond coated TKW 3-tip. 


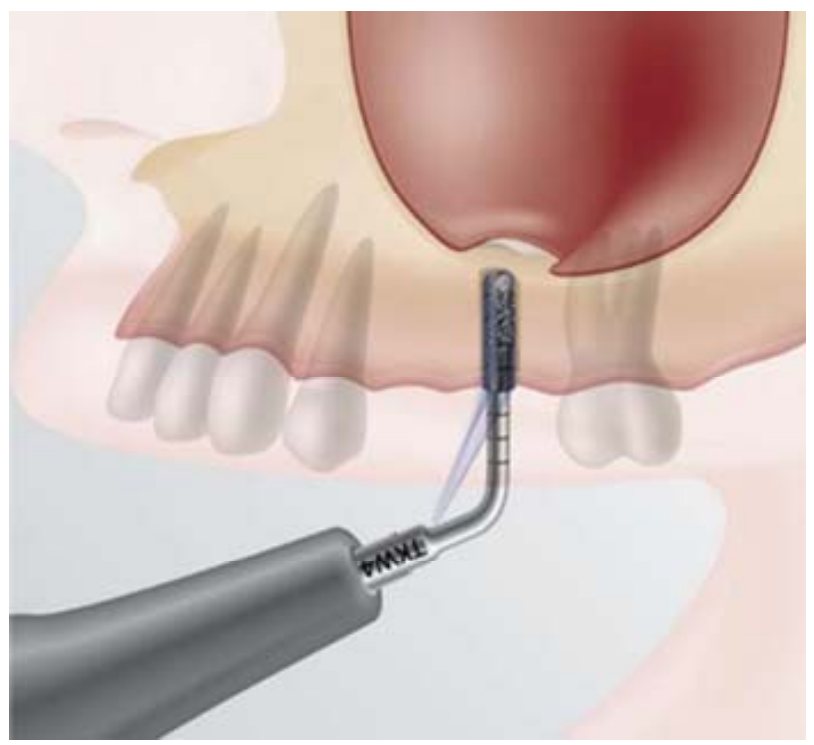

Figure 10. Final widening and smoothing of the transcrestal canal to $3.0 \mathrm{~mm}$ diameter for smooth application of bone graft.

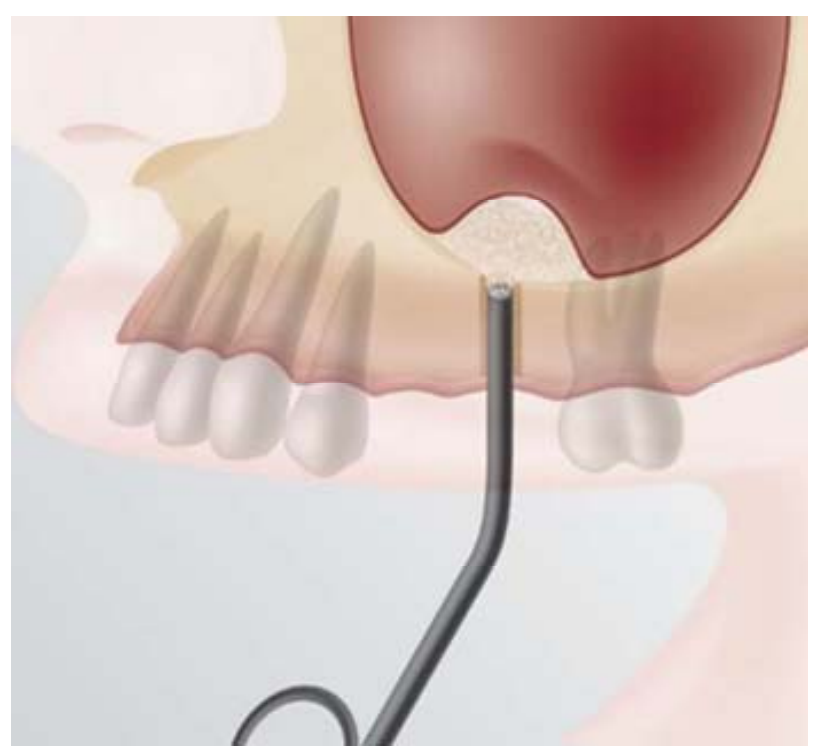

Figure 11. Application of bone graft with a common bone applicator. The amount of bonegraft applied depends on the necessary extension of the subantral augmentation.

type, prior maxillary sinus surgery on sinuslift side $y / n$, chronic sinusitis $\mathrm{y} / \mathrm{n}$, general medication (type);

Amount of anesthetic applied in ml, crestal mucoperiostal flap approach or gingiva-punch (f/p), mucoperiostal thickness at approach site in $\mathrm{mm}$, dental socket number of INTRALIFT-approach-site, real subantral alveolar crest height measured intraoperatively, surgeon's subjective rating of bone quality (D1-4);

Perforation of the sinus membrane detected $y / n$, commercial name of inserted bone graft, amount of bone graft inserted in $\mathrm{ccm}$, resulting subantral augmentation height in panoramic x-ray/CAT-scan/CBCT, additional

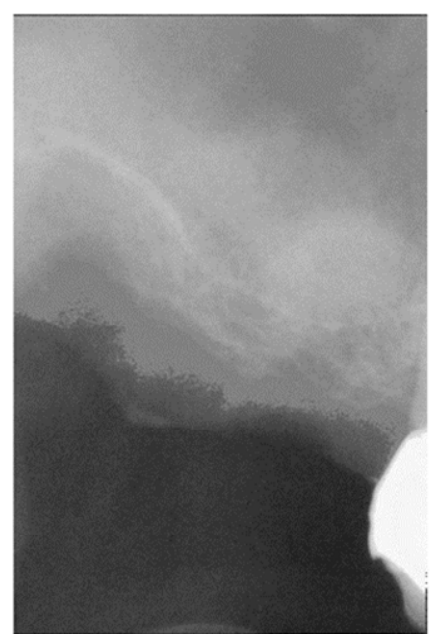

Figure 12. Intrasurgical $x$-ray check of augmentation extension: $0.5 \mathrm{ml}$ bone graft applied.

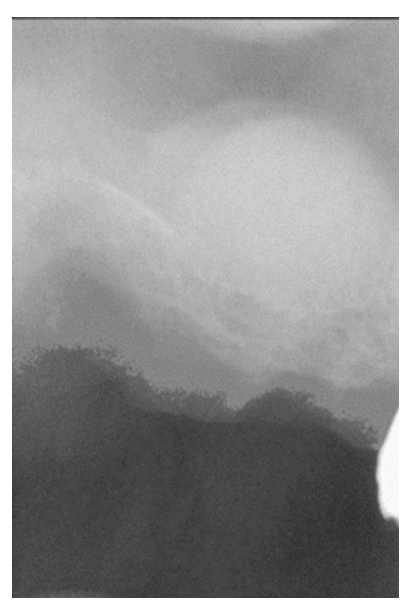

Figure 13. Intrasurgical x-ray check of augmentation extension: $1 \mathrm{ml}$ bone graft applied.

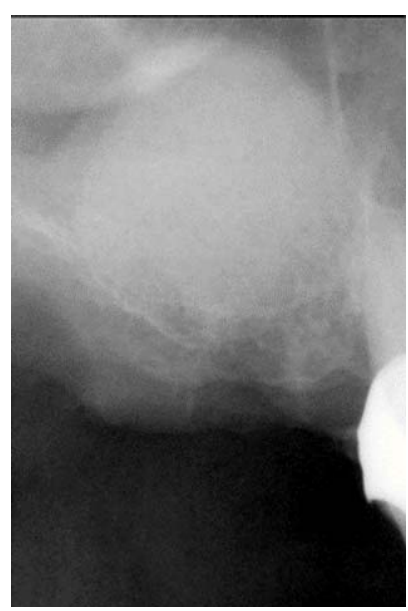

Figure 14. Intrasurgical x-ray check of augmentation extension: $2 \mathrm{ml}$ bone graft applied. 
surgical procedures;

Noticeable swelling two, three and seven days post surgery $y / n$ and in case yes the severity (no swelling of lower eyelid but only the cheek, swelling of lower eyelid, swelling of lower AND upper eyelid), subjective patient's report on pain after 7 days, analgetic medication applied (generic substance), number of analgetic tablets consumed by the patient in the first 7 days;

Occurance of complications $\mathrm{y} / \mathrm{n}$, type of complication, failure of tHUCSL-INTRALIFT (post surgical removal of bone graft);

Immediate implant insertion, delayed implant insertion date, bone quality at delayed implant insertion, implant position(s), implant type/manufacturer, implant dimensions length/diameter, Immediate loading $\mathrm{y} / \mathrm{n}$, insertion torque at implant insertion, date of implant loading, type of prosthetic treatment, in case of implant loss: date, follow up investigation 3, 6, 9, 12 and 24 months.

Follow up investigations included inspection of the INTRALIFT-surgical site and/or inserted implant(s), calibrated panoramic x-ray radiography, percussion test of the implant(s) to verify osseointegration state and/or prosthetic suprastructure, subjective condition of the patient (any inconvenience: $\mathrm{y} / \mathrm{n}$ ).

For statistical evaluation the surgeons name was anonymized by assigning a number $(1-6)$ as well as the patients names by assigning running numbers from 1 - 412 with 457 tHUCSL-INTRALIFT surgeries. Statistical evaluation was performed with mean-value denomination, standard deviation and p-test. Cases were only excluded from statistical evaluation when critical data were missing such as procedure related nominations and follow ups.

\section{RESULTS}

\subsection{Comparison of Success-Rates among Participating Surgeons}

Six surgeons treated 412 patients in 457 maxillary sinus sites with the tHUCSL-INTRALIFT-procedure. 8 patients and 11 INTRALIFT-sites-although successfulhad to be excluded from evaluation due to substantial lack of documentation. 404 fully documented patients with 446 tHUCSL-INTRALIFT sites were treated with 637 implants of different brands with an overall failure rate of $2.69 \%$.

No significant difference ( $p>0.53$ ) in the overall failure rates between the six surgeons could be observed (Table 1).

\subsection{Patient Age and Sex Distribution, General/Local Health State and Smoking Habit}

The average patient's age was 52 yrs starting from 19 yrs up to an age of 79 yrs, sex distribution resulted in $64.1 \%$ female and $35.9 \%$ male patients, 3 patients were missing age documentation (Table 2)

144 patients were smokers (35.64\%; 10 - 20 cigarettes per day), 252 non-smokers (62.38\%), 8 patients were not documented for smoking anamnesis (1.98\%). 23 patients (5.7\%) suffered from diabetes but had permission for elective surgical interventions by their General Physicians. 28 patients (6.93\%) underwent minor surgeries of their maxillary sinuses in their medical histories (mostly apisectomy related interventions in the 2nd premolar or 1st molar region, opened sinuses after tooth extraction, minor ENT-interventions) and 48 patients (11.88\%) were on permanent medication, mostly for heart diseases, blood pressure and mild anticoagulation (salicylic acid $50 \mathrm{mg}$ or $100 \mathrm{mg} / \mathrm{day}$ ).

\subsection{Amount of Local Anesthetic Applied}

The mean amount of the standard local anesthetic Articain (Ultracain dental/Ultracain dental forte) applied per tHUCSL-INTRALIFT-site-surgery was $4 \mathrm{ml}$ (min. 1.00 ml, max. $8.00 \mathrm{ml}$, Stand. Dev. 1.26), 8 patients were treated with INTRALIFT in general anesthesia.

Table 1. number of patients per surgeon ${ }^{1}$, tHUCSL-procedures per surgeon ${ }^{2}$, inserted implants per surgeon ${ }^{3}$, case exclusions due to lack of documentation ${ }^{4}$ and failure rates per surgeon ${ }^{5}$.

\begin{tabular}{|c|c|c|c|c|c|}
\hline & $\begin{array}{l}{ }^{1} \text { Fully doc } \\
\text { Patient \# }\end{array}$ & ${ }^{2}$ tHUCSL-sites & ${ }^{3}$ Inserted implants & $\begin{array}{c}{ }^{4} \text { Exclusions (Pat/OPsite } \\
\text { not doc suff) }\end{array}$ & $\begin{array}{l}{ }^{5} \text { Overall failure rate (count } \\
\text { per tHUCSL-sites/surgeon) }\end{array}$ \\
\hline surg 1 & 141 & 154 & 198 & $3 / 4$ & $5(3.25 \%)$ \\
\hline surg 2 & 102 & 109 & 148 & $2 / 2$ & $2(1.84 \%)$ \\
\hline surg 3 & 97 & 113 & 173 & $2 / 3$ & $3(2.65 \%)$ \\
\hline surg 4 & 38 & 40 & 71 & $1 / 2$ & $1(2.50 \%)$ \\
\hline surg 5 & 16 & 18 & 28 & $0 / 0$ & $1(5.56 \%)$ \\
\hline surg 6 & 10 & 12 & 19 & $0 / 0$ & $0(0.00 \%)$ \\
\hline \multirow[t]{2}{*}{ Total } & 404 & 446 & 637 & $8 / 11$ & $12(2.69 \%)$ \\
\hline & & & Significance: & $\begin{array}{l}\text { Failure rate difference } \\
\text { between surgeons }\end{array}$ & $(\mathrm{p}>0.53)$ \\
\hline
\end{tabular}


Table 2. Age $^{1}$ and $\operatorname{sex}^{2}$ distribution of patients treated with tHUCSL.

\begin{tabular}{ccc}
\hline Age Mean Value $^{1}$ & 52 & Number of females $^{2}$ \\
\hline Age Max & 79 & 259 \\
Age Min & 19 & $64.1 \%$ \\
under 30a & 12 & \\
under 40a & 70 & Number of males ${ }^{2}$ \\
under 50a & 102 & 145 \\
under 60a & 104 & \\
over 60a & 113 & 404 \\
Total & 401 & \\
missing age doc & 3 & \\
\hline
\end{tabular}

\subsection{Types of Surgical Approach}

In 144 tHUCSL-sites the crestal gingiva was opened by a $6 \mathrm{~mm}$ gingival punch (32.29\%), in 300 cases by a top crestal mucoperiostal flap with a size between $8 \times 8 \mathrm{~mm}$ to $10 \times 10 \mathrm{~mm}(67.26 \%)$. 2 cases $(0.45 \%)$ were not documented for the gingival approach (flap or punch). The gingiva thicknesses measured intraoperatively varied from 2 to $5 \mathrm{~mm}$ with an average thickness of $3 \mathrm{~mm}$. (Stand. Dev. 0.69)

\subsection{Intrasurgical tHUCSL-INTRALIFT Sites Related Results}

The mean subantral alveolar crest height at the tHUCSLINTRALIFT-approach site measured intraoperatively and scaled to full mm-values was $4 \mathrm{~mm}$ starting at $1 \mathrm{~mm}$ up to a subantral alveolar crest height of $10 \mathrm{~mm}$ (Figure 15), the bone quality mostly D2, followed by D3 and only rarely D1 or D4.

The transcrestal approach sites were chosen case specific and by the extension/pneumatization of the maxillary sinus from the first premolar area to the 2nd upper molar area both as "single hole approach" (entire augmentation performed via a single transcrestal trepanation) or as a "dual hole approach" (augmentation performed via two transcrestal trepanations; Figure 16). One site was not documented exactly for approach location (Figure 17).

\subsection{Implant-Insertion Related Data (Single and Two Stage)}

A total of 637 implants were inserted into the augmented sites of which 302 implants (47.41\%) were inserted simultaneously after the tHUCSL-INTRALIFT and subantral augmentation and 335 implants (52.59\%) in a second stage surgery.

The mean value of time elapsed between tHUCSL and insertion of the 335 implants in a second stage surgery

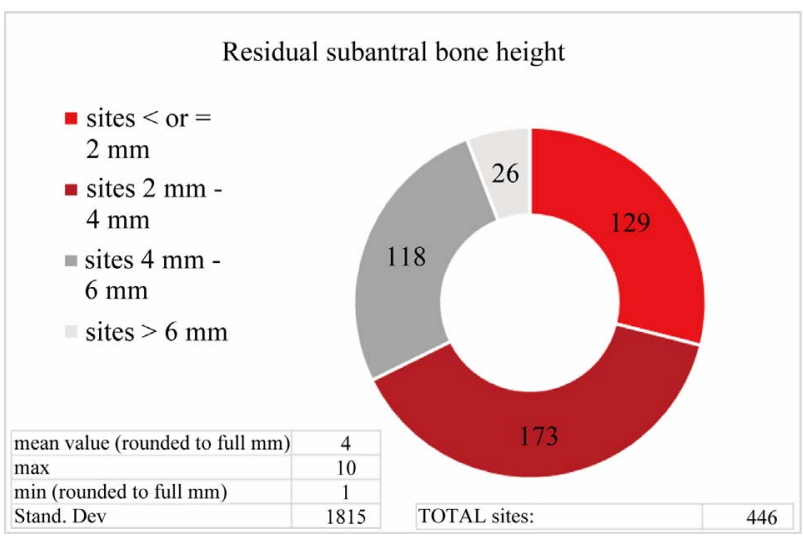

Figure 15. Intraoperatively measured heights of the subantral alveolar crest at the tHUCSL-INTRALIFT site.

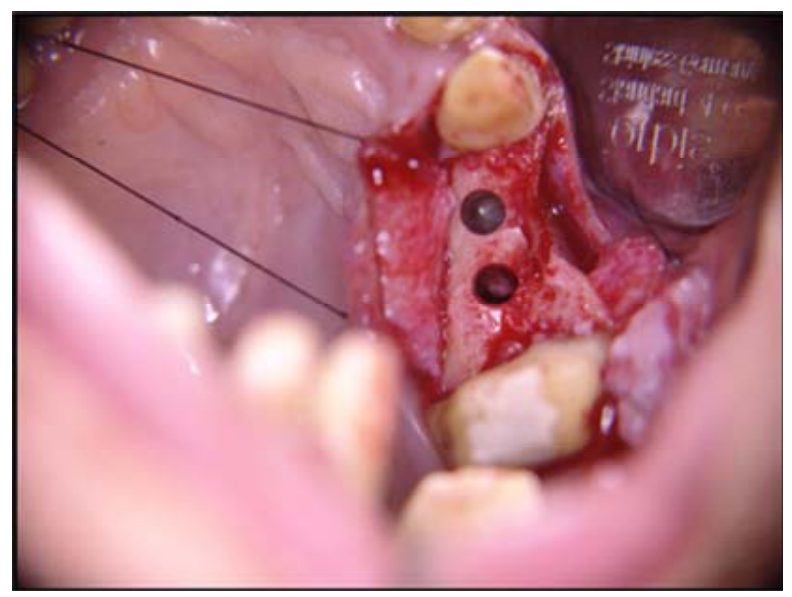

Figure 16. "Two hole approach" to sinus floor: membrane detachment is carried out first via one single hole approach. After membrane detachment a second trepanation is prepared for subantral filling of bone graft. The intact sinus-membrane is well visible.

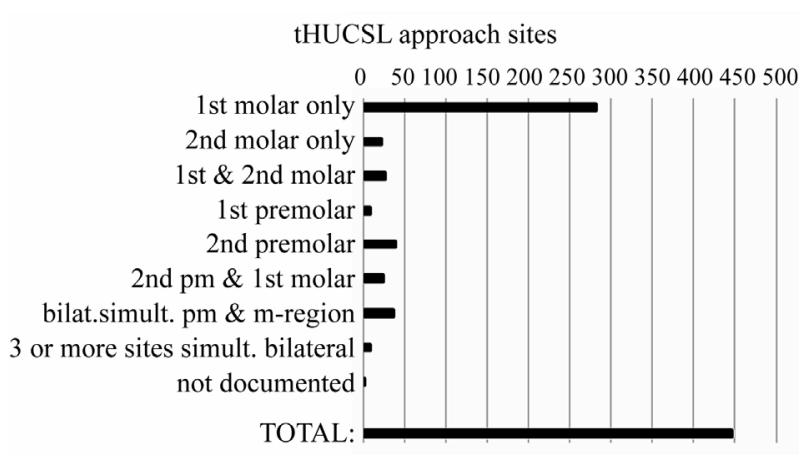

Figure 17. Denomination of tHUCSL subantral approach sites and number of transcrestal trepanations.

was 7.92 months (max. 16.80 months, min. 3, 72 months, Stand. Dev. 0.22) with 68 implants inserted after less than 6 month (20.3\%), 192 implants inserted after less than 9 months (57.31\%), and 75 implants (22.39\%) after more than 9 months. 


\section{7. tHUCSL-INTRALIFT Perforation Occurrence and Implant Loss Correlation}

In 25 sites (6\%) of all 446 tHUCSL-surgical sites the surgeon detected-visually or by Valsalva-test-a perforation of the sinus membrane. Nevertheless, all surgeons decided to proceed with subantral grafting backed by experimental suggestion [41] and only 2 implants (0.31\%) were lost in these cases with an insignificant correlation ( $p>0.83)$ compared to the overall failure rate.

\subsection{Grafting Materials and Achieved Augmentation Heights and Volumes}

As grafting materials “easygraft” (SUNSTAR-Degradable/CH), "Nanobone” (Artoss/GER), "BegoOss” (Bego/ Ger), "Cerasorb” (Curasan/CH), "BioOss” (Geistlich/CH) and other brands were applied (Figure 18).

The achieved average augmentation volume was 1.9 $\mathrm{ccm}$ starting at $0.4 \mathrm{ccm}$ up to $7.0 \mathrm{ccm}$ in very vast and highly pneumatized maxillary sinuses with an average achieved augmentation height of $10.7 \mathrm{~mm}$ starting at 4 mm height up to $17 \mathrm{~mm}$ (Figure 19). In 14 cases (3\%) autologous bone was added to the synthetic bone graft, in 432 cases (97\%) no autologous bone was added to the synthetic or xenogenic bone graft.

\subsection{Implant Brands, Implant Success-Rates and Prosthetic Treatment}

The 637 implants inserted were from different brands and manufacturers, the overall loss was 15 implants (2.35\%) in the 2-year survey-period with no significant difference in brands and manufacturers $(p>0.4)$ (Table 3).

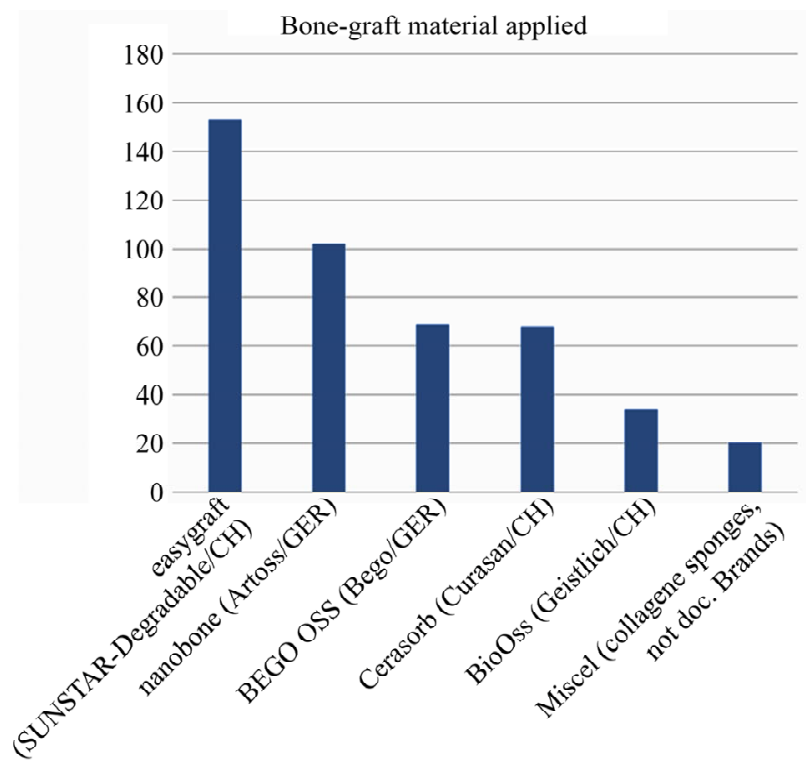

Figure 18. Bone grafting materials applied.
Achieved augmentation heights (mm)

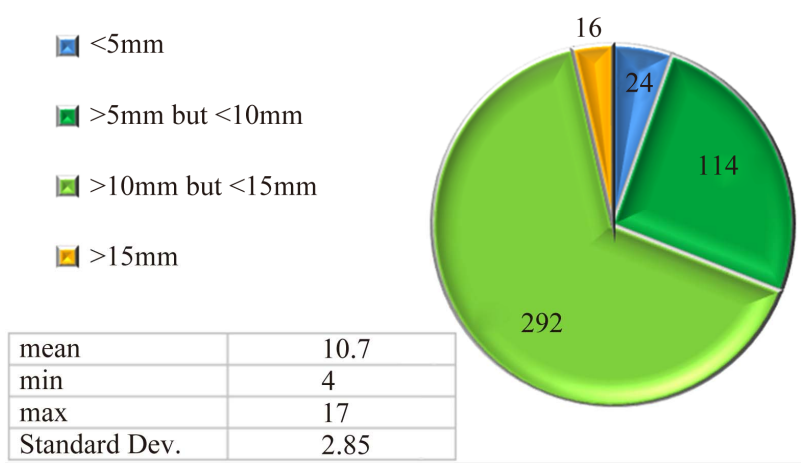

Figure 19. Achieved augmentation heights.

Table 3. Brands and implant types inserted into tHUCSL-INTRALIFT sites ${ }^{1}$ and brand related loss rates ${ }^{2}$.

\begin{tabular}{lc}
\hline${ }^{1}$ Implant Brands and Types & ${ }^{2}$ Implants lost per brand \\
\hline Q2/QK (Trinon GmbH) & $6(2.24 \%)$ \\
268 & \\
3 i Certain/Nanotite (BIOMET 3i) & $3(3.09 \%)$ \\
97 & \\
BEGO S/RI (BEGO) & $2(2.38 \%)$ \\
84 & \\
Alphatech (Alphatech) & $2(2.41 \%)$ \\
83 & \\
Ankylos (DentSply) & $1(1.34 \%)$ \\
75 & \\
Misc (Straumann. Nobel-Biocare. n/a etc.) & $1(3.33 \%)$ \\
30 & $15(2.35 \%)$ \\
Total: 637 & $(\mathrm{p}>0.4)$ \\
Significance &
\end{tabular}

The loading of implants and prosthetic treatment took place after a mean period of 8.6 month (max. 18 months, min. 5.76 months, Stand. Dev. 0.28) after implant insertion both for single stage as well as two-stage implant insertions.

623 implants out of 637 inserted implants were eligible for prosthetic treatment starting after less than 6 months (145 implants; 23.27\%) with smaller augmentations of less than $1 \mathrm{ccm}$, between 6 and 9 months (344 implants; 55.22\%) with average augmentation volumina of $2 \mathrm{ccm}$, up to a prosthetic treatment after more than 9 months (134 implants, 21.51\%) when augmentation volumina were greater than $2 \mathrm{ccm}$ and/or unforeseen causes delayed the prosthetic treatment. Unforeseen causes delaying the prosthetic treatment after a 12 month healing period were-according to written comments by the participating surgeons-mostly:

Acute general deseases of patients older than 65 yrs (acute cardial, gastrointestinal, gynocological, skeletal 
deseases) preventing prosthetic treatment due to general desease-specific rehabilitation, financial reasons (jobloss/savings-loss due to general economic state) and in some cases organizational delays on patient's or surgeon's side (job related, pregnancy etc.).

Overall implant loss before prosthetic treatment was 14 implants (2.2\%) of which 12 implants (1.88\%) showed no stable osseointegration (dull percussion sound, no rotational stability and visible micromovement) and had to be removed. Two simultaneously inserted implants $(0.31 \%)$ were lost due to postsurgical infectious complications, one implant $(0.16 \%)$ was lost 1 year after loading within the observation period due to periimplantitis.

\subsection{Patient tHUCSL-Intralift Related Morbidity Data}

344 patients (85\%) received a peri- or postsurgical antibiotic shielding with Clindamycin or Amoxicillin for 3 5 days, 60 patients (15\%) were not shielded with an antibiotic. A highly significant $(* * *)$ number of this patientgroup (84\%) suffered from postsurgical infectious complications whereas only $8 \%$ suffered from postsurgical infection when shielded with an antibiotic ( $p=0.001)$.

Overall postsurgical complications occurred in 3\%:

$84 \%$ of all complications were related to post-surgical infections in the patient group that was not shielded with an antibiotic. One patient suffered from a postsurgical infection although shielded with an antibiotic. One patient lost the bone graft transnasal with an accompanying stronger bleeding maybe caused by the intake of an unknown dosage of Acetyl Salicylic Acid the evening before surgery which the patient did not report to the surgeon upon inquiry.

97\% of all patients did not suffer from any postsurgical complication.

The average postsurgical analgetic intake (ibuprofen/ dexibuprofen $400 \mathrm{mg}$ ) was 1.7 tablets with a maximum of 12 tablets and a minimum of zero tablets in the first 14 days after surgery (Stand. Dev. 0.43). Postsurgical pain also showed a seasonal distribution: in winter, early spring and late autumn exogenic acquired secondary viral infections such as colds led to a higher consumption of analgetics than in the rest of the year $(\mathrm{p}<0.05)$.

Postsurgical visible swelling on day two, three and seven post tHUCSL was reported for 58 patients (14\%) of which 49 patients (84\%) showed only mild swelling (no edema of the corresponding lower lid), 8 patients (14\%) medium swelling (edema of lower eyelid) and 1 patient (2\%) strong swelling with edema also of the upper eyelid on the surgery-side. 346 patients (86\%) were reported free of visible swelling (Figures 20-22).

All patients were asked to score their subjective im-

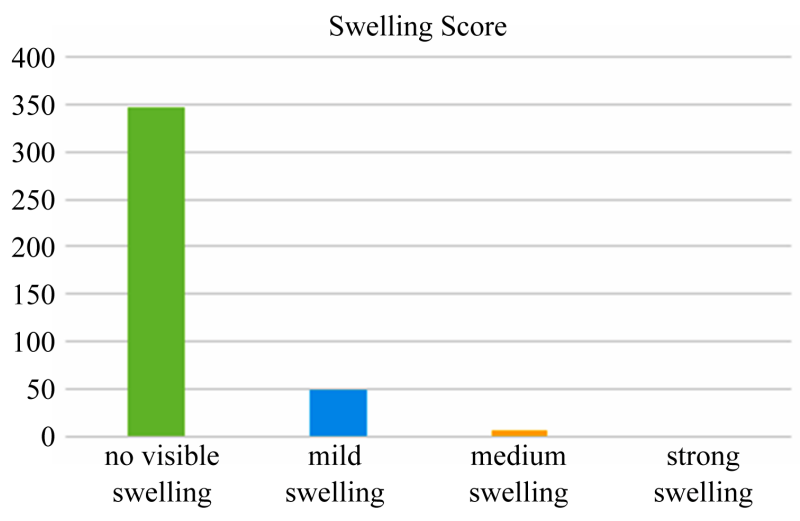

Figure 20. Swelling score.

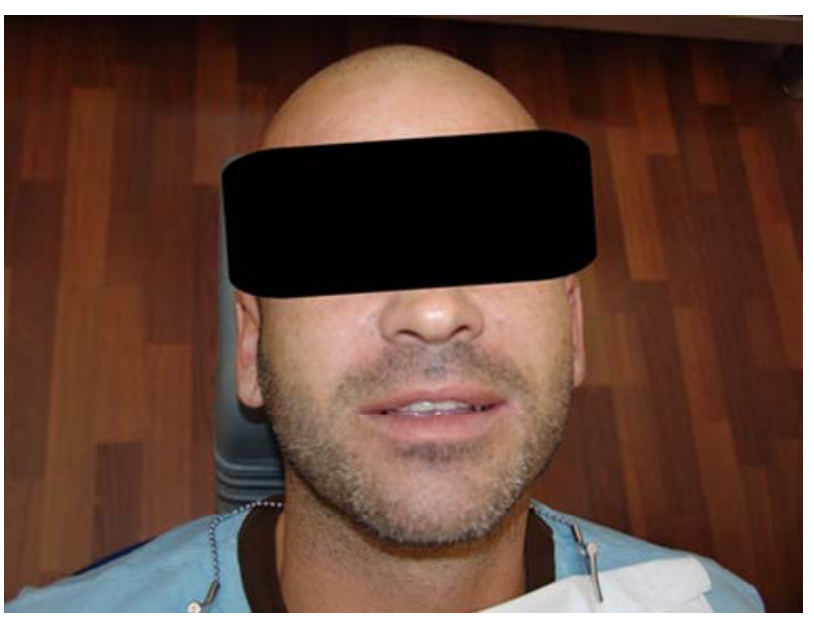

Figure 21. Patient case I: facial situation 2 days after INTRALIFT in the right maxillary sinus: no swelling can be detected.

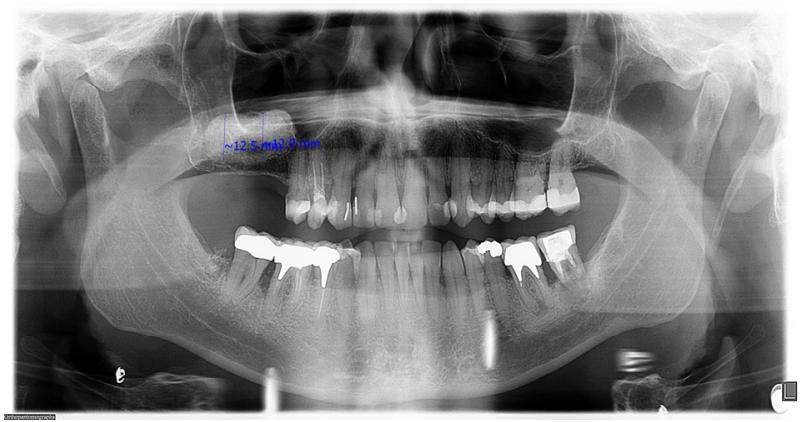

Figure 22. Patient case I: post surgical X-ray of the patient in Figure 16 with a substantial subantral augmentation with easygraft (SUN-STAR-Degradable/CH).

pression of postsurgical pain: $13 \%$ declared to have experienced postsurgical pain, $87 \%$ declared not to have experienced any postsurgical pain (Figure 23).

\section{DISCUSSION}

The overall results of this study suggest the tHUCSLINTRALIFT to be a minimal-invasive alternative not 


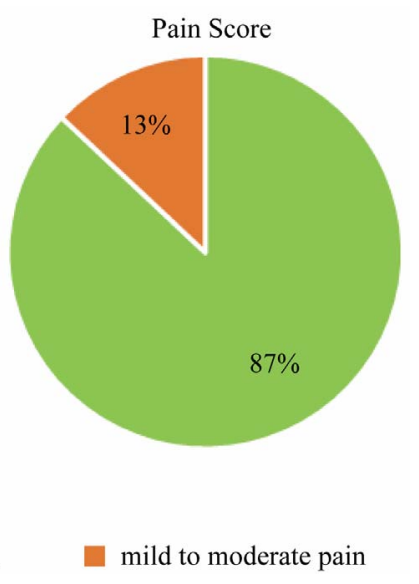

Figure 23. Pain score.

only to traditional transcrestal sinuslift techniques but also to lateral approach sinuslift-procedures and is applicable to all common sinuslift indications. In cases of narrow interdental gaps-when other transcrestal sinuslift-techniques cannot be applied due to lack of approximal space-it revealed it's applicability as an alternative to lateral approach sinuslift-techniques.

The achieved augmentation volumina with a mean value of $1.9 \mathrm{ccm}$ correspond to results achievable with the lateral approach sinuslift technique if necessary but inhere the minimal invasiveness of transcrestal procedures.

The risk of sinus-membrane perforation is low with $6 \%$ compared to lateral approach sinus-lift procedures performed with rotating instruments with a reported perforation rate of $14 \%$ - 56\% [42] due to the use of diamond coated ultrasonic instruments which are known to be highly safe on soft tissues [43-46]. Since all surgeons —backed by experimental results published [41]—decided to proceed with subantral grafting even in these reported cases of sinus-membrane-perforation, only 2 implants $(0.31 \%)$ were lost in these cases, suggesting a perforation as described by Jank et al. [41] not to be a reason to interrupt the procedure.

The preparation of the transcrestal approach and the hydrodynamic detachment of the sinus-membrane grants a minimum risk of procedural perforations and seems to avoid complete ruptures of the membrane in the detachment process since the surgeon need not touch the sinus membrane with instruments [36].

The standardized hydrodynamic pressure described in the surgical protocol [38] combined with the ultrasound cavitation effect distributes the detaching forces equally between the sinus membrane and the bony antrum of the sinus. The tHUCSL-INTRALIFT thus excludes the highest risk-factor in the detachment process-the surgeons hand [27-33] — by the non-contact procedure and stan- dardized and calibrated detachment forces provided by the Piezotome-device.

The tHUCSL-INTRALIFT-procedure can be trained with a small investment of time by the dentist and be applied by every dentist with a basic training in implanttology with almost the same success-rate as long-term experienced oral surgeons as the study results suggest. It is applicable to every anatomical situation (Figures 24-32) and achieves an equal overall success rate as

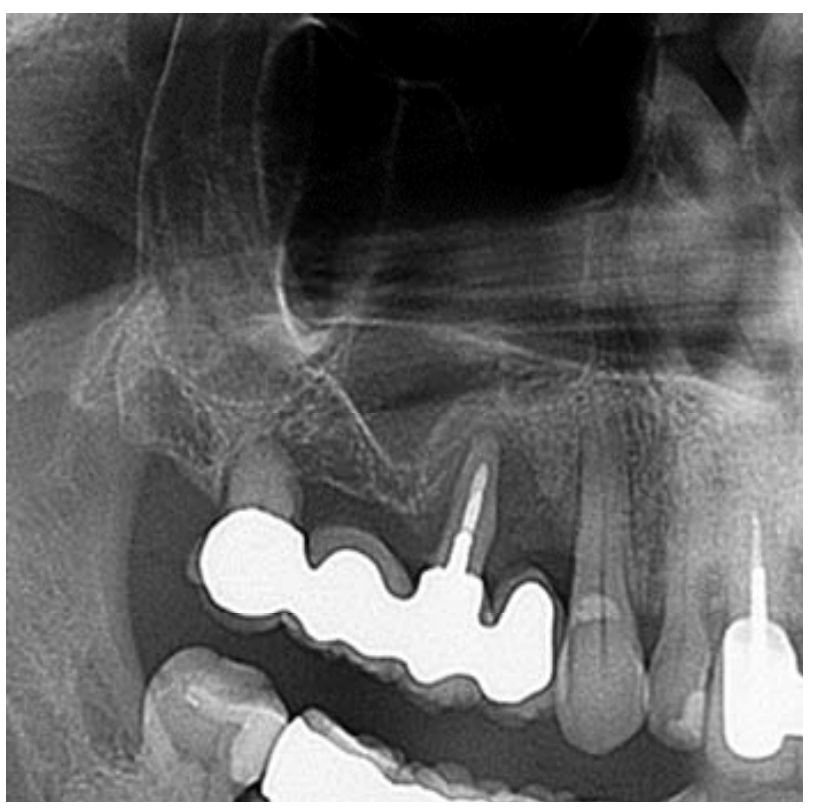

Figure 24. Patient case II: presurgical situation.

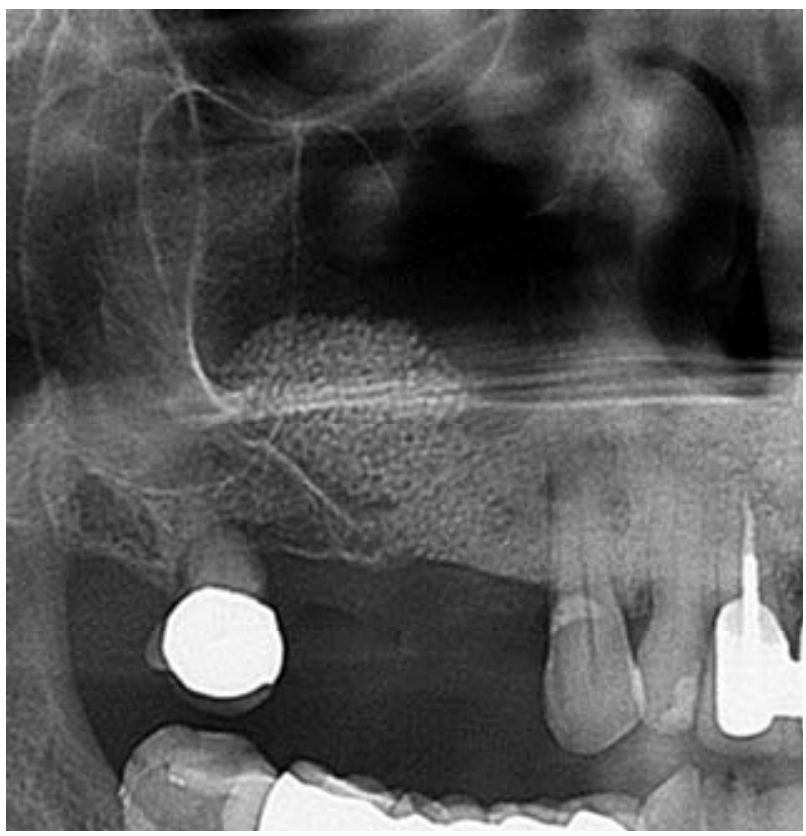

Figure 25. Patient case II: situation 6 month post INTRALIFT with easygraft (SUNSTAR-Degradable/CH) prior to implant insertion of 2 Q2-implants (Trinon/GER). 


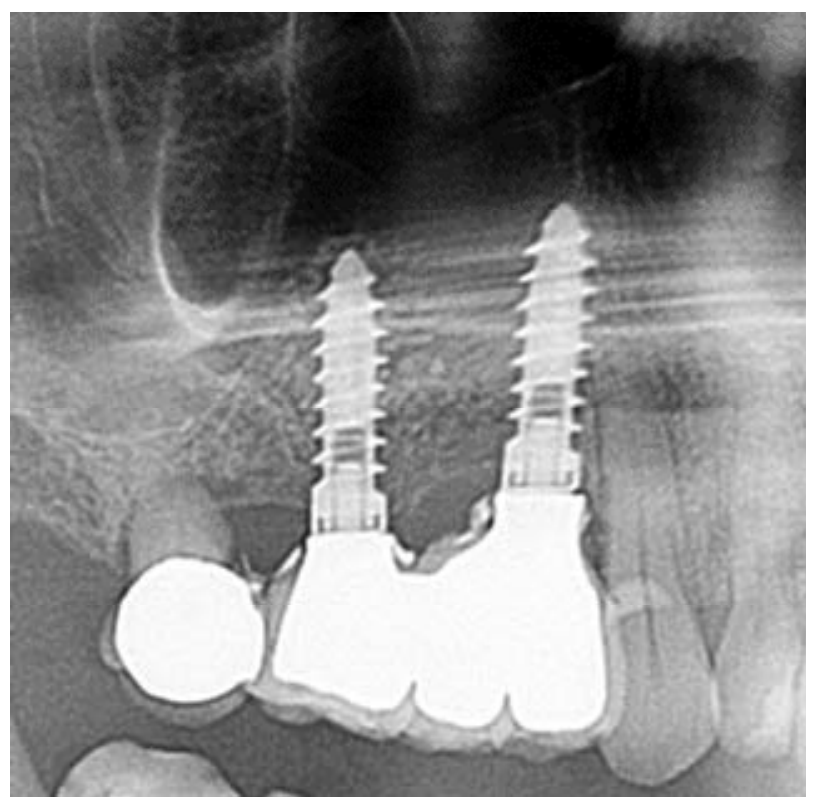

Figure 26. Patient case II: final prosthetic treatment after 8.5 months.

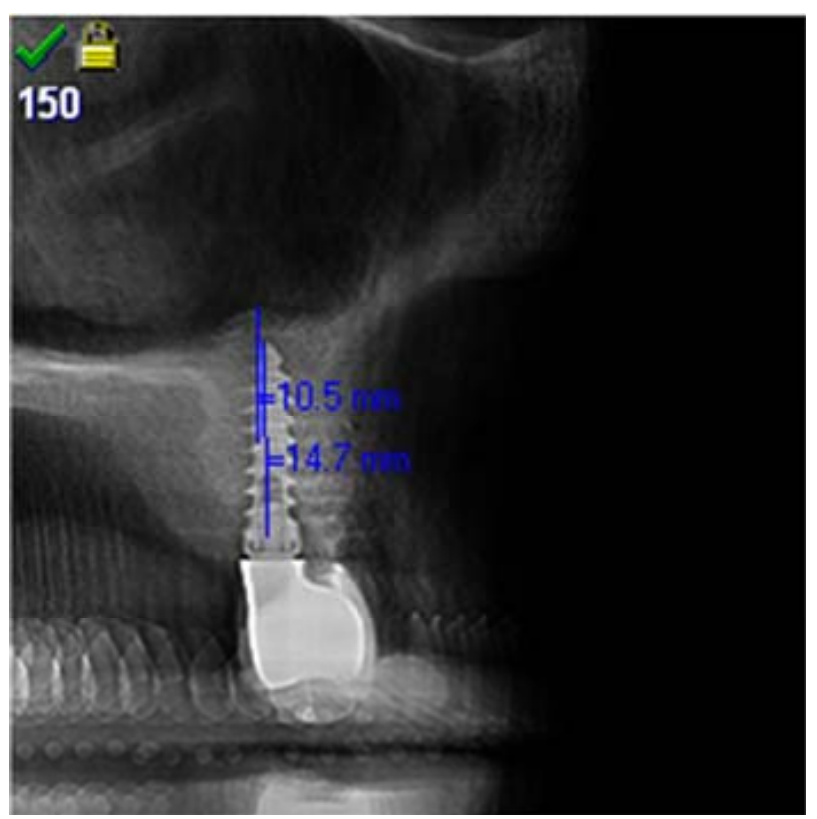

Figure 27. Patient case III: INTRALIFT with Nanobone (Artoss/GER) simultaneous single tooth implant insertion (Q2-Implant, Trinon) and prosthetic loading after 5 months.

other transcrestal sinuslift techniques [47-49] but is not limited by a minimum subantral alveolar crest height and achievable augmentation volumina. With tHUCSL the indication for a transcrestal approach can be extended compared to traditional transcrestal procedures and applied to indications which were reserved for lateral approach sinuslift-procedures until now.

Patients experience very little postsurgical pain and swelling and mostly are able to work within 3 - 5 days

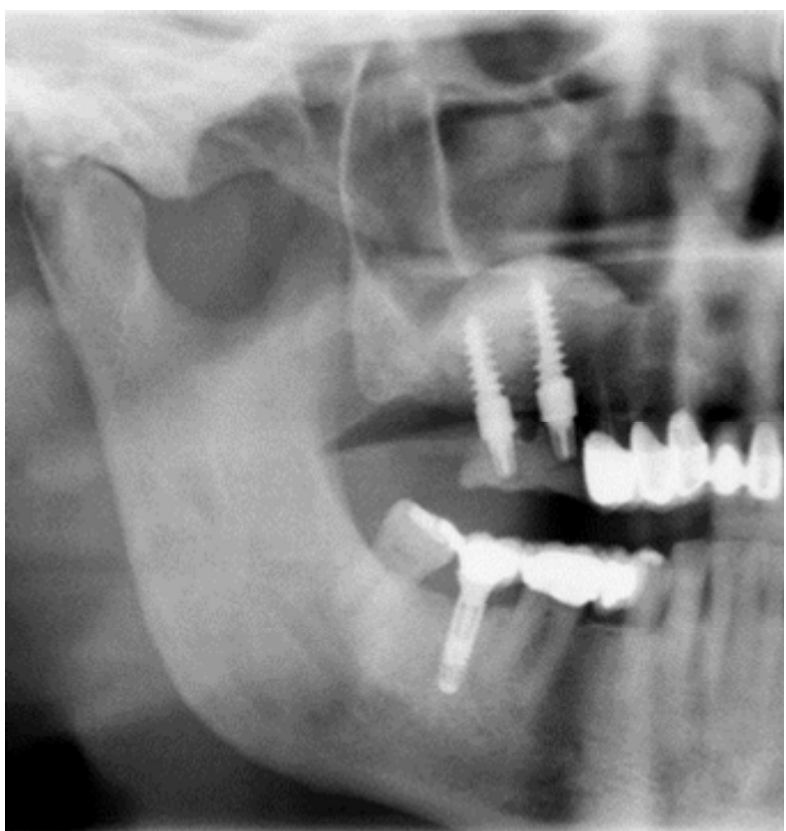

Figure 28. Patient case IV: INTRALIFT with "easygraft" (SUNSTAR-Degradable/CH) and simultaneous insertion of 2 single-stage Q1-implants (Trinon/GER) and pseudo-immediate-loading (infraocclusal resin provisional).

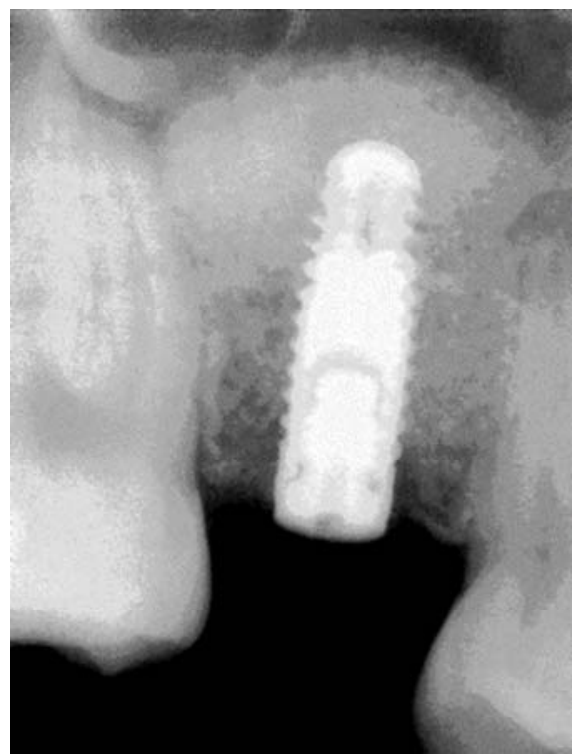

Figure 29. Patient case V: INTRALIFT with BEGO-OSS (BEGO/GER) and simultaneous implant insertion (IMZ-Implant, DentsplyFriadent/USA) in a narrow dental gap (4 $\mathrm{mm})$.

after surgery and thus accept sinus lifting to a higher degree.

The overall implant-loss rate of $2.35 \%$ is not related to the experience of the dentist and complications are mostly related to postsurgical infections when no antibiotic shielding is prescribed. In the rare case of a complete 


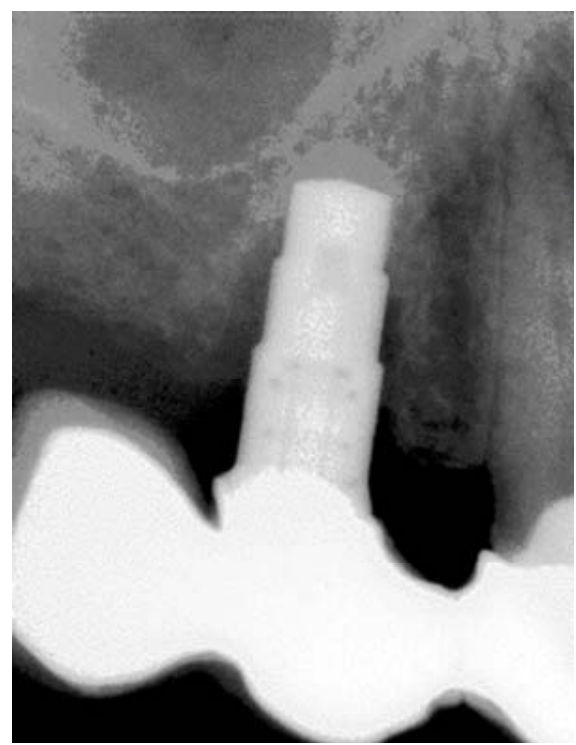

Figure 30. Patient case VI: loosened old dental implant has to be removed.

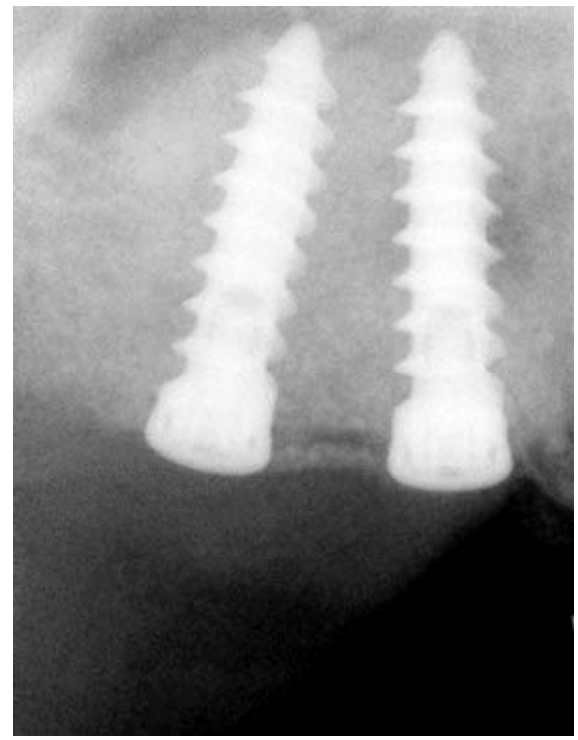

Figure 31. Patient case VI: immediately after implant removal INTRALIFT was carried out with Nanobone (Artoss/GER) and simultaneous implant insertion of 2 Q2-implants (Trinon/GER).

rupture of the sinus-membrane or loss of the bone graft the procedure did not cause major inconveniences on the patient's side and can be repeated after 3 - 5 months.

The success-rate of the tHUCSL-INTRALIFT is independent from the type and brand of used bone-graftmaterial as well as the type and brand of inserted implants as already generally described by Del Fabbro et al. for transcrestal sinus-floor elevation in a systematic review [47].

Especially in older patients the tHUCSL-INTRALIFT

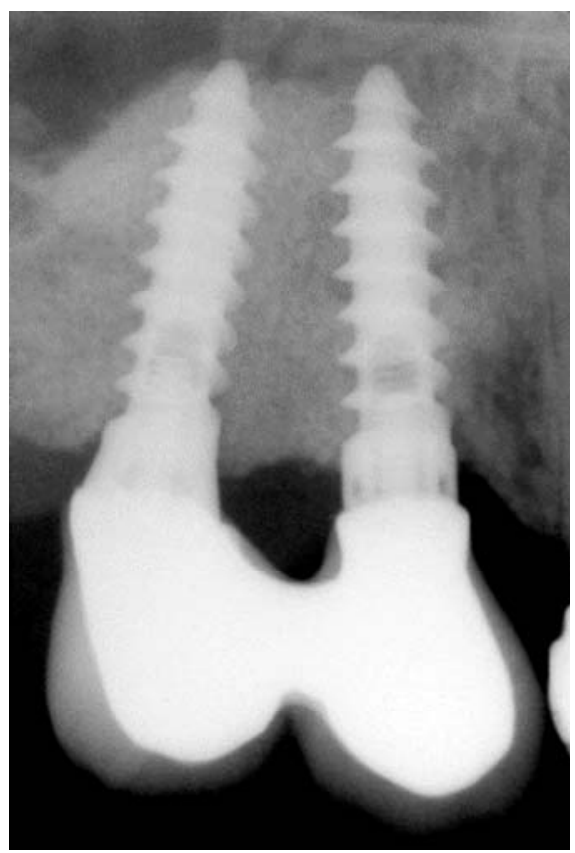

Figure 32. Patient case VI: prosthetic treatment after 7 months.

enables simultaneous bilateral augmentations of the sinus-floor without major surgical trauma thus widening the indication spectrum to a wider and older base of patients with remaining subantral alveolar crest heights of 1 $\mathrm{mm}$ or less and proves the osteogenic capacity of the atraumatically detached sinus membrane independent from age [50] (Figures 33-36).

The indication for a sinuslift procedure might be questionable at subantral crest heights of $8 \mathrm{~mm}$ or more since a high reliability of short implants is discussed. Nevertheless, the results of this study clearly show that two thirds of patients in need of implants in the lateral maxilla presented equal or less than $4 \mathrm{~mm}$ subantral alveolar crest-height thus being not eligible for treatment with short implants (Figure 15).

\section{CONCLUSION}

The tHUCSL-INTRALIFT is compatible to all implantsystems with an implant - diameter of more than $3 \mathrm{~mm}$ and-most of all-applicable to all anatomical conditions of the alveolar crest and the maxillary sinus which can be considered a major advantage. Compared to other transcrestal instrument sets, balloon- or hydraulic-assisted sinuslift systems or "sinuslift"-implants that demand a minimum mesio-distal distance of the dentition gap and/or a subantral alveolar crest height of more than $4 \mathrm{~mm}$, the results of this prospective study suggest the tHUCSL-INTRALIFT to be a safe and predictable alternative also to lateral-approach sinuslift-techniques. It can be applied beginning with maxillary dentition gaps of a 


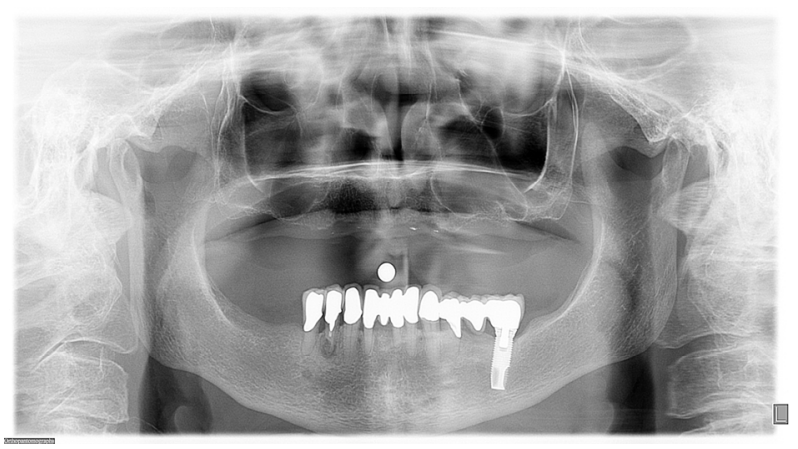

Figure 33. Patient case VII: 78 yrs old female with edentulous maxilla after bilateral INTRALIFT and augmentation with collagenous sponges (2.5 ccm each side).

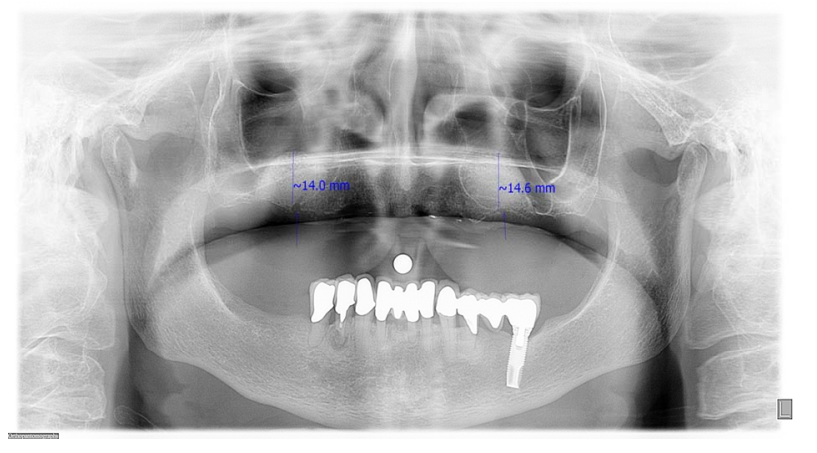

Figure 34. Patient case VII: panoramic X-ray 7 months post INTRALIFT (blue arrows mark the approach-osteotomy site).

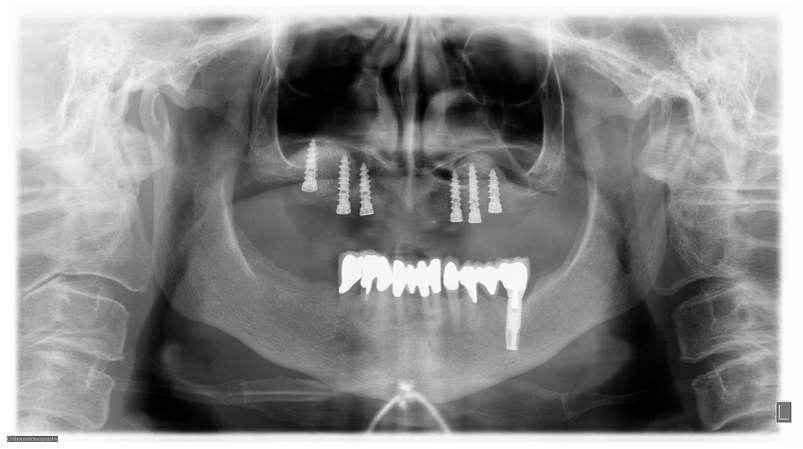

Figure 35. Patient case VII: insertion of 6 Q2-implants (Trinon/GER) 7 months after bilateral INTRALIFT.

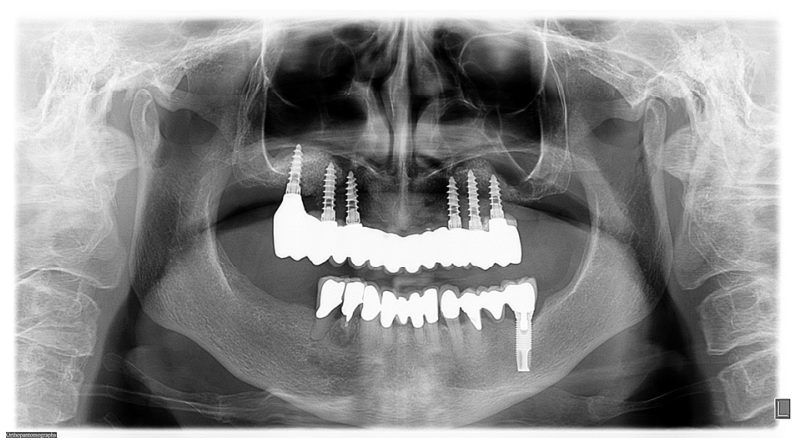

Figure 36. Patient case VII: loading of implants 11 months after INTRALIFT with a full arch Zirkonia-bridge. minimum of $3.5 \mathrm{~mm}$ up to the entire edentulous maxilla with remaining subantral alveolar crest heights of $1 \mathrm{~mm}$ or less. Thus it might fulfil the demand of the ever growing community of dentists confronted with the demand of a highly safe and minimal invasive sinuslift-procedure in the growing number of cases with little or no remnant subantral alveolar crest and highly pneumatised maxillary sinuses with the least risk of failure and procedural patient morbidity.

\section{REFERENCES}

[1] Boyne, P. and James, R.A. (1980) Grafting of the maxillary sinus floor with autogenous marrow and bone. Journal of Oral and Maxillofacial Surgery, 17, 113-116.

[2] Tatum, O.H. (1986) Maxillary and sinus implant reconstruction. Dental Clinics of North America, 30, 207-229.

[3] Chanavaz, M. (1990) Maxillary sinus: Anatomy, physicology, surgery and bone grafting related to implantology. Eleven years of surgical experience (1979-1990). Journal of Oral Implantology, 16, 199-209.

[4] Summers, R.B. (1994) A new concept in maxillary implant surgery: The osteotome technique. Compendium of Continuing Education in Dentistry, 15, 152-162.

[5] Summers, R.B. (1998) Sinus floor elevation with osteotomes. Journal of Esthetic and Restorative Dentistry, 10, 164-171. http://dx.doi.org/10.1111/j.1708-8240.1998.tb00352.x

[6] Zitzmann, N.U. and Scharer, P. (1998) Sinus elevation procedures in the resorbed posterior maxilla: Comparison of the crestal and lateral approaches. Oral Surgery, Oral Medicine, Oral Pathology, Oral Radiology and Endodontology, 85, 8-17. http://dx.doi.org/10.1016/S1079-2104(98)90391-2

[7] Raghoebar, G.M., Timmenga, N.M., et al. (2001) Maxillary bone grafting for insertion of endosseous implants: results after 12 - 124 months. Clinical Oral Implants Research, 12, 279-286. http://dx.doi.org/10.1034/j.1600-0501.2001.012003279.x

[8] Kahnberg, K.E., Ekestubbe, A., et al. (2001) Sinus lifting procedure. I. One-stage surgery with bone transplant and implants. Clinical Oral Implants Research, 12, 479-487. http://dx.doi.org/10.1034/j.1600-0501.2001.120508.x

[9] Marx, R.E. and Garg, A.K. (2002) A novel aid to elevation of the sinus membrane for the sinus lift procedure. Implant Dentistry, 11, 268-271. http://dx.doi.org/10.1097/00008505-200207000-00012

[10] Engelke, E., Schlegel, A., et al. (2002) The endoscopically controlled osteotome sinus floor elevation: A preliminary prospective study. International Journal of Oral \& Maxillofacial Implants, 17, 557-566.

[11] Smiler, D.G. and Holmes, R.E. (1987) Sinus lift procedure using porous hydroxyapatite: A preliminary clinical report. Journal of Oral Implantology, 13, 239-253.

[12] Block, M.S. and Kent, J.N. (1997) Sinus augmentation for dental implants: The use of autogenous bone. Journal of Oral and Maxillofacial Surgery, 55, 1281-1286. 
http://dx.doi.org/10.1016/S0278-2391(97)90185-3

[13] Van den Bergh, J.P.A., Ten Bruggenkate, C.M., et al. (2000) Anatomical aspects of sinus floor elevations. Clinical Oral Implants Research, 11, 256-265. http://dx.doi.org/10.1034/j.1600-0501.2000.011003256.x

[14] Cordioli, G., Mazzocco, C., et al. (2001) Maxillary sinus floor augmentation using bioactive glass granules and autogenous bone with simultaneous implant placement. Clinical and histological findings. Clinical Oral Implants Research, 12, 270-278.

http://dx.doi.org/10.1034/j.1600-0501.2001.012003270.x

[15] Cammack, G., Nevins, M., et al. (2005) Histologic evaluation of mineralized and demineralized freeze-dried bone allograft for ridge and sinus augmentations. International Journal of Periodontics and Restorative Dentistry, 25, 231-237.

[16] Zijderveld, S.A., Zerbo, I.R., et al. (2005) Maxillary sinus floor augmentation using a beta-tricalcium phosphate (Cerasorb) alone compared to autogenous bone grafts. International Journal of Oral \& Maxillofacial Implants, 20, 432-440.

[17] Szabo, G., et al. (2005) A prospective multicenter randomized clinical trial of autogenous bone versus betatricalcium phosphate graft alone for bilateral sinus elevation: Histologic and histomorphometric evaluation. International Journal of Oral \& Maxillofacial Implants, 20, 371-381.

[18] Boyne, P.J., Lilly, L.C., et al. (2005) De novo bone induction by recombinant human bone morphogenetic protein-2 (rhBMP-2) in maxillary sinus floor augmentation. Journal of Oral and Maxillofacial Surgery, 63, 1693-707. http://dx.doi.org/10.1016/j.joms.2005.08.018

[19] Kim, Y.-K., Yun, P.-Y., et al. (2009) Analysis of the healing process in sinus bone grafting using various grafting materials. Oral Surgery, Oral Medicine, Oral Pathology, Oral Radiology and Endodontology, 107, 204-211. http://dx.doi.org/10.1016/j.tripleo.2008.07.021

[20] Wallace, S.S. and Froum, S.J. (2003) Effect of maxillary sinus augmentation on the survival of endosseous dental implants. A systematic review. Annals of Periodontology, 8, 328-343. http://dx.doi.org/10.1902/annals.2003.8.1.328

[21] Wilk, R.M. (1999) Physiology of the maxillary sinus. Oral \& Maxillofacial Surgery Clinics of North America, 11, 15-19.

[22] Solar, P., Geyerhofer, U., et al. (1999) Blood supply to the maxillary sinus relevant to sinus floor elevation procedures. Clinical Oral Implants Research, 10, 34-44. http://dx.doi.org/10.1034/j.1600-0501.1999.100105.x

[23] Lundgren, S., Anderson, S., et al. (2004) Bone reformation with sinus membrane elevation: A new surgical technique for maxillary sinus floor augmentation. Clinical Implant Dentistry and Related Research, 6, 165-173.

[24] Celik D, Turan T, Kabukcuoglu F, et al. (2003) Bone induction capacity of the periosteum and neonatal dura in the setting of the zygomatic arch fracture model. Archives of Facial Plastic Surgery, 5, 301-308. http://dx.doi.org/10.1001/archfaci.5.4.301

[25] Ortak, T.O., et al. (2005) Osteogenic Capacities of periost grafts, periost flaps and prefabricated periosteal flaps: Experimental study. Journal of Craniofacial Surgery, 16, 594-600. http://dx.doi.org/10.1097/01.scs.0000168773.71356.62

[26] Srouji, S., Kizhner, T., et al. (2009) The schneiderian membrane contains osteoprogenitor cells: In vivo and in vitro study. Calcified Tissue International, 84, 138-145. http://dx.doi.org/10.1007/s00223-008-9202-x

[27] Hernández-Alfaro, F., Torradeflot, M.M., et al. (2008) Prevalence and management of Schneiderian membrane perforations during sinus-lift procedures. Clinical Oral Implants Research, 19, 91-98.

[28] Greenstein, G., Cavallaro, J., et al. (2008) Clinical recommendations for avoiding and managing surgical complications associated with implant dentistry: A review. Journal of Periodontology, 79, 1317-1329. http://dx.doi.org/10.1902/jop.2008.070067

[29] Anavi, Y., Allon, D.M., et al. (2008) Complications of maxillary sinus augmentations in a selective series of patients. Oral Surgery, Oral Medicine, Oral Pathology, Oral Radiology and Endodontology, 106, 34-38. http://dx.doi.org/10.1016/j.tripleo.2007.09.021

[30] Li, J. and Wang, H.L. (2008) Common implant-related advanced bone grafting complications: Classification, etiology, and management. Implant Dentistry, 17, 389-401. http://dx.doi.org/10.1097/ID.0b013e31818c4992

[31] Katranji, A., Fotek, P., et al. (2008) Sinus augmentation complications: Etiology and treatment. Implant Dentistry, 17, 339-349. http://dx.doi.org/10.1097/ID.0b013e3181815660

[32] Jerjes, W., Upile, T., et al. (2010) Experience in third molar surgery: An update. British Dental Journal, 209, E1. http://dx.doi.org/10.1038/sj.bdj.2010.581

[33] Waseem, J., Mohammed, E., et al. (2006) Experience versus complication rate in third molar surgery. Head \& Face Medicine, 2, 14. http://dx.doi.org/10.1186/1746-160X-2-14

[34] Vercellotti, T., De Paoli, S., et al. (2001) The piezoelectric bony window osteotomy and sinus membrane elevation: Introduction of a new technique for simplification of the sinus augmentation procedure. International Journal of Periodontics and Restorative Dentistry, 21, 561-567.

[35] Kfir, E., Kfir, V., et al. (2006) Minimally invasive antral membrane balloon elevation followed by maxillary bone augmentation and implant fixation. Journal of Oral Implantology, 32, 26-33. http://dx.doi.org/10.1563/782.1

[36] Vitkov, L., Gellrich, N.C., et al. (2005) Sinus floor elevation via hydraulic detachment and elevation of the Schneiderian membrane. Clinical Oral Implants Research, 16, 615-621. http://dx.doi.org/10.1111/j.1600-0501.2005.01161.x

[37] Sotirakis, E.G. and Gonshor, A. (2005) Elevation of the maxillary sinus floor with hydraulic pressure. Journal of Oral Implantology, 31, 197-204.

http://dx.doi.org/10.1563/1548-1336(2005)31[197:EOTM SF]2.0.CO;2

[38] Troedhan, A.C., Kurrek, A., et al. (2010) Hydrodynamic ultrasonic sinus floor elevation-An experimental study 
in sheep. Journal of Oral and Maxillofacial Surgery, 68, 1125-1130. http://dx.doi.org/10.1016/j.joms.2009.12.014

[39] Velázquez-Cayón, R., Romero-Ruiz, M.M., et al. (2012) Hydrodynamic ultrasonic maxillary sinus lift: Review of a new technique and presentation of a clinical case. Medicina Oral Patologia Oral y Cirugia Bucal, 17, e271e275. http://dx.doi.org/10.4317/medoral.17430

[40] Wainwright, M., Troedhan, A., et al. (2007) The IntraLift: A new minimal invasive ultrasonic technique for sinus grafting procedures. Implants, 3, 30-34.

[41] Jank, S., Kurrek, A., et al. (2011) Rupture length of the sinus membrane after $1.2 \mathrm{~mm}$ puncture and surgical sinus elevation: An experimental animal cadaver study. Oral Surgery, Oral Medicine, Oral Pathology, Oral Radiology and Endodontology, 112, 568-572. http://dx.doi.org/10.1016/j.tripleo.2010.11.014

[42] Wallace, S.S., Mazor, Z., et al. (2007) Schneiderian membrane perforation rate during sinus elevation using piezosurgery: Clinical results of 100 consecutive cases. International Journal of Periodontics and Restorative Dentistry, 27, 413-419.

[43] Schaeren, S., Jaquiery, C., et al. (2008) Assessment of nerve damage using a novel ultrasonic device for bone cutting. Journal of Oral and Maxillofacial Surgery, 66, 593. http://dx.doi.org/10.1016/j.joms.2007.03.025

[44] Pavlíková, G., Foltán, R., et al. (2011) Piezosurgery in oral and maxillofacial surgery. International Journal of Oral and Maxillofacial Surgery, 40, 451-457. http://dx.doi.org/10.1016/j.ijom.2010.11.013
[45] Schlee, M., Steigmann, M., et al. (2006) Piezosurgery: basics and possibilities. Implant Dentistry, 15, 334-340. http://dx.doi.org/10.1097/01.id.0000247859.86693.ef

[46] Seshan, H., Konuganti, K. and Zope, S. (2009) Piezosurgery in periodontology and oral implantology. Journal of Indian Society of Periodontology, 13, 155-156. http://dx.doi.org/10.4103/0972-124X.60229

[47] Del Fabbro, M., Corbella, S., et al. (2012) Implant survival rates after osteotome-mediated maxillary sinus augmentation: A systematic review. Clinical Implant Dentistry and Related Research, 14, e159-e168. http://dx.doi.org/10.1111/j.1708-8208.2011.00399.x

[48] Yeong-Wook, K., Yoon-Seon, K., et al. (2010) A retrospective study of trans-crestal approach to maxillary sinus floor-elevation and simultaneous implant placement. Journal of the Korean Academy of Implant Dentistry, 29, 1-9.

[49] Tan, W.C., Lang, N.P., et al. (2008) A systematic review of the success of sinus floor elevation and survival of implants inserted in combination with sinus floor elevation Part II: Transalveolar technique. Journal of Clinical Periodontology, 35, 241-254.

[50] Troedhan, A., Kurrek, A., et al. (2012) Biological principles and physiology of bone regeneration under the Schneiderian membrane after sinus lift surgery: A radiological study in 14 patients treated with the transcrestal hydrodynamic ultrasonic cavitational sinus lift (intralift). International Journal of Dentistry, 2012, Article ID: 576238. 